\title{
Siklos waves with torsion in 3D
}

\section{Blagojević and B. Cvetković}

Institute of Physics, University of Belgrade, Pregrevica 118, 11080 Belgrade-Zemun, Serbia

E-mail: mb@ipb.ac.rs, cbranislav@ipb.ac.rs

ABSTRACT: Starting from the Siklos waves in general relativity with a cosmological constant, interpreted as gravitational waves on the anti-de Sitter background, a new class of exact torsion waves is constructed in the framework of three-dimensional gravity with propagating torsion. In the asymptotic limit, the geometry of torsion waves takes the antide Sitter form. In the sector with massless torsion modes, we found a set of asymptotic conditions that leads to the conformal asymptotic symmetry.

KEYwords: Classical Theories of Gravity, Field Theories in Lower Dimensions, Conformal and W Symmetry

ARXIV EPRINT: 1410.0800 


\section{Contents}

1 Introduction 1

2 Siklos waves 3

3 Siklos waves with torsion 4

3.1 Ansatz 4

3.2 Lagrangian dynamics of PGT 5

3.3 Solutions 6

4 Asymptotic conditions $\quad 7$

$\begin{array}{lll}4.1 & \text { Massive torsion waves } & 7\end{array}$

4.2 Massless torsion waves 8

5 Canonical form of the asymptotic symmetry 9

6 Concluding remarks 11

$\begin{array}{ll}\text { A AdS and Siklos spacetimes in 3D } & 11\end{array}$

B Improving the canonical generator $\quad 13$

\section{Introduction}

Exact gravitational waves have been an important subject of investigation in general relativity (GR) from the early 1920s; for a review, see [1-4]. Most of the activity on the subject has been focused on asymptotically flat models, the solutions of GR without a cosmological constant. From 1980s, exact gravitational waves have been studied also in GR with a cosmological constant $\left(\mathrm{GR}_{\Lambda}\right)$ [5-7], see also [8]; for higher-dimensional extensions, see [9-13]. In particular, exact gravitational waves with an AdS asymptotic behavior attracted a lot of interest in regard to the AdS/CFT correspondence [14, 15]. Moreover, some of these solutions "may serve as exact models of the propagation of primordial gravitational waves and may be relevant for the (hypothetical) cosmological wave background" [16].

To properly understand dynamical complexities of gravity, one often relies on technically simplified three-dimensional (3D) models (for a review and an extensive list of references, see $[17,18])$. In 3D, both $\mathrm{GR}$ and $\mathrm{GR}_{\Lambda}$ are topological theories without propagating degrees of freedom, in which nontrivial wave solutions can exist only in the presence of matter sources [19-21]. To avoid such a degenerate situation, one is naturally motivated to study alternative gravitational models possessing true dynamical degrees of freedom. The well-known models of this type, formulated in the context of Riemannian geometry of 
spacetime, are Topological massive gravity and New massive gravity [22-24]. Their dynamical properties allow for the existence of gravitational waves in vacuum; see, for instance, Ayón-Beato et al. [25].

In the early 1960s, a new approach to gravitational dynamics was proposed, based on a modern, gauge-field-theoretic approach, known as the Poincaré gauge theory (PGT) (see [26, 27], for a textbook exposition of PGT, [28] for an up-to-date status of PGT, including its 3D version, and [29]) with an underlying Riemann-Cartan (RC) geometry of spacetime, characterized by both the curvature and the torsion. In a topological version of the three-dimensional PGT, gravitational waves with torsion were constructed in the presence of matter sources by Obukhov [30]. However, genuine gravitational waves are those that can propagate in spacetime regions without matter. Further investigations of the PGT, with a Lagrangian that is at most quadratic in the field strengths (quadratic PGT), revealed a rich dynamical structure, expressed, in particular, by the existence of propagating torsion modes [31]. In a recent paper [32], ${ }^{1}$ we used quadratic PGT to construct exact torsion waves in vacuum as a generalization of the plane-fronted waves from GR.

In the present paper, we continue the investigation of genuine gravitational waves with torsion in 3D, by focusing on the anti-de Sitter (AdS) background. We found a new class of exact torsion waves in vacuum, representing a PGT extension of the Siklos waves in $\mathrm{GR}_{\Lambda}[33]$, see also $[8,16]$. In the linear approximation, this class is associated to spin-2 torsion excitations around the AdS background. In the sector of massless torsion modes, we found a set of asymptotic conditions that leads to a conformal asymptotic symmetry, characterized by two independent Virasoro algebras with central charges. On the other hand, massive torsion waves show kind of an oscillatory behavior in the asymptotic region.

The paper is organized as follows. In section 2, we give an overview of the Siklos waves in the three-dimensional $\mathrm{GR}_{\Lambda}$. In section 3 , we construct a new wave solution in PGT, taking the metric to be of the Siklos form, whereas the torsion piece of the connection is assumed to possess only the tensorial irreducible component. The solutions of the field equations are found and classified according to the values of the mass parameter $\mu^{2}$, associated to the spin-2 torsion modes. For $\mu^{2} \geq 0$ (no tachyons), the asymptotic limit of the Siklos waves with torsion is shown to be represented by Riemannian AdS spacetimes. In section 4 , we study the form of the AdS asymptotic conditions for $\mu^{2} \geq 0$. It turns out that a well-defined asymptotic structure exists only in the massless sector. The corresponding central charges of the asymptotic symmetry are found in section 5 , and section 6 is devoted to concluding remarks. Finally, two appendices contain some technical details.

Here are our conventions: the Latin indices $(i, j, k, \ldots)$ refer to the local Lorentz (co)frame and run over $(+,-, 2), b^{i}$ is the triad field (coframe 1 -form), $h_{i}$ is the dual basis (frame), totally antisymmetric tensor $\varepsilon^{i j k}$ is normalized to $\varepsilon^{+-2}=1$; the Greek indices $(\mu, \nu, \rho, \ldots)$ refer to the coordinate frame; the Lie dual of an antisymmetric form $X^{j k}$ is $X_{i}:=-\varepsilon_{i j k} X^{j k} / 2$, the Hodge dual of a form $\alpha$ is ${ }^{\star} \alpha$, and the exterior product of forms is implicit.

\footnotetext{
${ }^{1}$ Here, the reader can find references of earlier studies of exact gravitational waves with torsion in 4D.
} 


\section{Siklos waves}

In 1980s, Siklos [33] found a special class of exact gravitational waves propagating on the AdS background, the physical interpretation of which was investigated in detail by Podolský [16]. In the Poincaré coordinates $x^{\mu}=(u, v, y)$, the Siklos metric in 3D has the form

$$
d s^{2}=\frac{\ell^{2}}{y^{2}}\left[2 d u(H d u+d v)-d y^{2}\right],
$$

with $H=H(u, y)$, which is equivalent to a subclass of the Kundt metric $[8,16]$. The wave fronts are labeled by $u=$ const., $v$ is an affine parameter along the corresponding rays generated by the Killing vector field $\partial_{v}$ that is null but not covariantly constant, and for $H=0$ the metric reduces to the AdS background (see appendix A). We choose the triad field $b^{i}$ (1-form) to be

$$
b^{+}:=\frac{\ell}{y} d u, \quad b^{-}:=\frac{\ell}{y}(H d u+d v), \quad b^{2}=\frac{\ell}{y} d y,
$$

so that the line element is given by $d s^{2}=\eta_{i j} b^{i} b^{j}$, with the half-null Lorentz metric

$$
\eta_{i j}=\left(\begin{array}{ccc}
0 & 1 & 0 \\
1 & 0 & 0 \\
0 & 0 & -1
\end{array}\right) \text {. }
$$

The dual frame basis $h_{i}$, defined by $\left.h_{i}\right\lrcorner b^{j}=\delta_{i}^{j}$, is given by

$$
h_{+}=\frac{y}{\ell}\left(\partial_{u}-H \partial_{v}\right), \quad h_{-}=\frac{y}{\ell} \partial_{v}, \quad h_{2}=\frac{y}{\ell} \partial_{y} .
$$

The related Riemannian connection $\omega^{i j}$ (1-form) can be written in a compact form as

$$
\omega^{i j}=\bar{\omega}^{i j}-\frac{1}{\ell} \varepsilon^{i j} k^{m}\left(y H^{\prime}\right) k_{n} b^{n} .
$$

Here, prime denotes a derivative with respect to $y$, the first term $\bar{\omega}^{i j}$ describes the background AdS geometry,

$$
\bar{\omega}^{+-}=0, \quad \bar{\omega}^{+2}=\frac{1}{\ell} b^{+}, \quad \bar{\omega}^{-2}=\frac{1}{\ell} b^{-},
$$

and the second one is the radiation piece, characterized by the null vector $k^{m}:=(0,1,0)$, with $k_{m}=(1,0,0)$.

Next, we calculate the Riemannian curvature,

$$
R^{i j}=\frac{1}{\ell^{2}} b^{i} b^{j}-\frac{1}{\ell^{2}} \varepsilon^{i j}{ }_{m} k^{m}\left(y^{2} H^{\prime \prime}-y H^{\prime}\right) k^{n \star} b_{n},
$$

whereupon the Ricci curvature $\left.(R i c)^{i}=-h_{j}\right\lrcorner R^{i j}$ and the scalar curvature $\left.R=h_{i}\right\lrcorner(R i c)^{i}$ are found to be

$$
\begin{aligned}
(R i c)^{i} & =\frac{2}{\ell^{2}} b^{i}+\frac{1}{\ell^{2}} k^{i}\left(y^{2} H^{\prime \prime}-y H^{\prime}\right) k_{n} b^{n}, \\
R & =\frac{6}{\ell^{2}} .
\end{aligned}
$$


When the Siklos metric satisfies the vacuum field equation of $\mathrm{GR}_{\Lambda}$ with $\Lambda \sim-1 / \ell^{2}$, the metric function $H$ takes a simple form:

$$
y^{2} H^{\prime \prime}-y H^{\prime}=0 \quad \Rightarrow \quad H=D_{1}(u)+D_{2}(u) y^{2} .
$$

However, this solution is trivial. Indeed, since the radiation piece of the curvature vanishes on shell, we have $R^{i j}=b^{i} b^{j} / \ell^{2}$, and the geometry of spacetime is fixed, it has the AdS form. Nontrivial AdS waves can exist in $\mathrm{GR}_{\Lambda}$ only in the presence of matter [19-21], but to have vacuum AdS waves, one has to change the gravitational dynamics. As we shall see, transition to quadratic PGT allows the existence of genuine AdS waves with torsion.

\section{Siklos waves with torsion}

Basic gravitational variables of PGT are the triad field $b^{i}$ and the Lorentz connection $\omega^{i j}$ (1-forms), and the related field strengths are the torsion $T^{i}=d b^{i}+\omega^{i}{ }_{m} b^{m}$ and the curvature $R^{i j}=d \omega^{i j}+\omega_{m}^{i} \omega^{m j}$ (2-forms). Relying on PGT, we now introduce a geometric extension of the Siklos waves (2.1) to genuine Siklos waves with torsion.

\subsection{Ansatz}

In order to preserve the radiation nature of the Siklos metric, we assume that the form of the triad field in PGT remains the same as in eq. (2.2). Essentially the same idea can be applied also to the connection [32]: starting from the Riemannian connection (2.3), we assume that the new, RC connection is given by

$$
\omega^{i j}=\bar{\omega}^{i j}-\frac{1}{\ell} \varepsilon^{i j}{ }_{m} k^{m}(y G) k_{n} b^{n}
$$

where

$$
G:=H^{\prime}+K, \quad K=K(u, y) .
$$

Geometrically, the new function $K$ in the connection is related to the torsion:

$$
T^{i}:=\nabla b^{i}=-\frac{y K}{\ell} k^{i} k^{n \star} b_{n} .
$$

For $K=0$, the torsion vanishes, and the connection becomes equivalent to $\bar{\omega}^{i j}$. The only nonvanishing irreducible component of $T^{i}$ is its tensorial piece ${ }^{(1)} T^{i}$ [32], so that

$$
{ }^{(1)} T^{i}=T^{i} .
$$

Using the above ansatz for the connection, one can calculate the $\mathrm{RC}$ curvatures:

$$
\begin{aligned}
R^{i j} & =\frac{1}{\ell^{2}} b^{i} b^{j}-\frac{1}{\ell^{2}} \varepsilon^{i j}{ }_{m} k^{m}\left(y^{2} G^{\prime}-y H^{\prime}\right) k^{n \star} b_{n}, \\
(R i c)^{i} & =\frac{2}{\ell^{2}} b^{i}+\frac{1}{\ell^{2}} k^{i}\left(y^{2} G^{\prime}-y H^{\prime}\right) k_{n} b^{n}, \\
R & =\frac{6}{\ell^{2}} .
\end{aligned}
$$


The quadratic curvature invariant takes the form

$$
R^{i j \star} R_{i j}=\frac{6}{\ell^{4}}{ }^{\star} 1 .
$$

The only nonvanishing irreducible components of $R^{i j}$ are:

$$
{ }^{(6)} R^{i j}=\frac{1}{6} R b^{i} b^{j}, \quad{ }^{(4)} R^{i j}=R^{i j}-{ }^{(6)} R^{i j} .
$$

For more details on the irreducible decomposition of the field strengths, see ref. [32].

In what follows, the specific forms of both the metric function $H$ and the torsion function $K$ will be determined by the PGT field equations.

\subsection{Lagrangian dynamics of PGT}

The PGT dynamics is described by a Lagrangian 3-form $L_{G}=L_{G}\left(b^{i}, T^{i}, R^{i j}\right)$, which is assumed to be at most quadratic in the field strengths (quadratic PGT) and parity invariant. In conformity with our ansatz, the Lagrangian is chosen to have the form

$$
\begin{aligned}
L_{G}= & -a_{0} \varepsilon_{i j k} b^{i} R^{j k}-\frac{1}{3} \Lambda_{0} \varepsilon_{i j k} b^{i} b^{j} b^{k} \\
& +T^{i \star}\left(a_{1}{ }^{(1)} T_{i}\right)+\frac{1}{2} R^{i j \star}\left(b_{4}{ }^{(4)} R^{i j}+b_{6}{ }^{\left({ }^{6}\right)} R^{i j}\right) .
\end{aligned}
$$

Indeed, the only nonvanishing irreducible components of the field strengths appearing in $L_{G}$ are ${ }^{(1)} T^{i},{ }^{(4)} R^{i j}$ and ${ }^{(6)} R^{i j}$, and $a_{1}, b_{4}, b_{6}$ are the corresponding coupling constants. Then, the PGT field equations in vacuum are found to be (see section III.A of ref. [32]):

$$
\begin{aligned}
& (1 S T): \quad\left(a_{0} \ell^{2}-b_{4}-b_{6}\right)\left(y H^{\prime \prime}-H^{\prime}\right)+\left(a_{0} \ell^{2}-a_{1} \ell^{2}-b_{4}-b_{6}\right) y K^{\prime}=0, \\
& 2 a_{0} \ell^{2}+b_{6}+2 \ell^{4} \Lambda_{0}=0 \\
& (2 N D): \quad b_{4}\left[y^{2}\left(H^{\prime \prime \prime}+K^{\prime \prime}\right)+y K^{\prime}\right]-\left(a_{0} \ell^{2}-a_{1} \ell^{2}-b_{6}\right) K=0 .
\end{aligned}
$$

These equations are checked using the Excalc package of the computer algebra system Reduce. Using the expression for $(1 S T)^{\prime}$, one finds that $(2 N D)$ can be rewritten as

$$
y^{2} K^{\prime \prime}+y K^{\prime}+\ell^{2} \mu^{2} K=0, \quad \mu^{2}=\frac{\left(a_{1}-a_{0}-b_{6} \lambda\right)\left(a_{0}+b_{4} \lambda+b_{6} \lambda\right)}{b_{4} a_{1}},
$$

where $\lambda:=-1 / \ell^{2}$. Finally, after introducing the notation

$$
\hat{y}=\frac{y}{\ell}, \quad m^{2}=\ell^{2} \mu^{2},
$$

the two field equations take a more compact form:

$$
\begin{aligned}
\hat{y} H^{\prime \prime}-H^{\prime} & =\ell C \hat{y} K^{\prime}, \quad C:=\frac{a_{1}}{a_{0}+b_{4} \lambda+b_{6} \lambda}-1, \\
\hat{y}^{2} K^{\prime \prime}+\hat{y} K^{\prime}+m^{2} K & =0
\end{aligned}
$$

where prime now denotes differentiation with respect to $\hat{y}$. As one can see, it is the presence of torsion $(K \neq 0)$ that makes the metric of the AdS wave nontrivial $\left(\hat{y} H^{\prime \prime}-H^{\prime} \neq 0\right)$. Equations (3.6) define a new class of Siklos waves - the Siklos waves with torsion. 


\subsection{Solutions}

The coefficient $m^{2}$ in $(2 N D)$ is the (dimensionless) mass parameter associated to the spin-2 excitation of the torsion field around the AdS background, see [31, 32]. The absence of tachyons requires $m^{2} \geq 0$. In this subsection, we construct the exact Sikos waves with torsion, and classify them according to the values of $m^{2}$.

(1) $\boldsymbol{m}^{\mathbf{2}}>\mathbf{0}$. The Euler (or Euler-Fuchs, Euler-Cauchy) differential equation $(2 N D)$ is solved by the ansatz $K=\hat{y}^{\alpha}$, which yields $\alpha^{2}+m^{2}=0$. For $m^{2}>0$, we have $\alpha= \pm i m$, so that $K=\hat{y}^{ \pm i m}=e^{ \pm i m \ln \hat{y}}$, or equivalently,

$$
K=A(u) \cos (m \ln \hat{y})+B(u) \sin (m \ln \hat{y}) .
$$

By substituting this result into $(1 S T)$, one finds the related solution for $H$ :

$$
H=D_{1}+D_{2} \hat{y}^{2}+\frac{\ell C m}{1+m^{2}} \hat{y}[A(u) \sin (m \ln \hat{y})-B(u) \cos (m \ln \hat{y})] .
$$

The first two terms, which represent a solution of the homogeneous equation $\hat{y} H^{\prime \prime}-H^{\prime}=0$, can be geometrically disregarded, as they do not influence the values of the field strengths.

In the asymptotic limit $\hat{y} \rightarrow 0$, the torsion and the radiation piece of the curvature, ${ }^{(4)} R_{i j}$, vanish, as follows from the relations

$$
\begin{aligned}
\lim _{\hat{y} \rightarrow 0} \hat{y} K & =0, \\
\lim _{\hat{y} \rightarrow 0}\left[\hat{y}^{2}\left(H^{\prime \prime}+K^{\prime}\right)-\hat{y} H^{\prime}\right]=\lim _{\hat{y} \rightarrow 0}\left[\hat{y}^{2} K^{\prime}+\ell C \hat{y}^{2} K^{\prime}\right] & =0 .
\end{aligned}
$$

Thus, the asymptotic geometry of our solution is given by the Riemannian AdS spacetime.

(2) $\boldsymbol{m}^{2}=\mathbf{0}$. In order to have a smooth Minkowskian limit for $\ell^{2} \rightarrow \infty$, the condition $m^{2}=0$ is realized by demanding [31]

$$
a_{1}-a_{0}+b_{6} / \ell^{2}=0 .
$$

As a consequence, the solution for the massless torsion wave is given by

$$
\begin{aligned}
& K=C_{1}+C_{2} \ln \hat{y}, \\
& H=D_{1}+D_{2} \hat{y}^{2}-\ell C C_{2} \hat{y} .
\end{aligned}
$$

As before, one can choose $D_{1}=D_{2}=0$ without loss of generality, so that the asymptotic limit of the solution is again given by the Riemannian AdS spacetime.

(3) $\mathbf{m}^{\mathbf{2}}<\mathbf{0}$. Although the spin-2 torsion modes are now tachyons, we present the related exact wave solution, for the sake of completeness:

$$
\begin{aligned}
K & =A \hat{y}^{m}+B \hat{y}^{-m}, \\
H & =\frac{\ell C m}{m^{2}-1}\left(A \hat{y}^{1+m}-B \hat{y}^{1-m}\right) .
\end{aligned}
$$

The asymptotic behavior depends on the value of $m$. 


\section{Asymptotic conditions}

In our study of the asymptotic conditions, we assume that the topology of the spacetime manifold $M$ is $R \times \Sigma$, where $R$ is interpreted as time, and $\Sigma$ is a spatial section of spacetime, whose boundary $\partial \Sigma$ is topologically a circle. The asymptotic analysis is simplified by introducing a new set of local coordinates $(t, \varphi)$, given by $u=(t+\ell \varphi) / \sqrt{2}, v=(t-\ell \varphi) / \sqrt{2}$, such that the boundary $\partial \Sigma$ at $y=0$ is parametrized by the angular coordinate $\varphi$.

As we have seen in the previous section, in the asymptotic limit $y \rightarrow 0$, the geometry of our torsion wave is described by the Riemannian AdS spacetime. This property motivates us to examine asymptotic conditions based on the following requirements:

(a) asymptotic configurations include the torsion wave geometry;

(b) they are invariant under the action of the AdS group $\mathrm{SO}(2,2)$;

(c) asymptotic symmetries have well defined canonical generators.

Specific aspects of these criteria depend on the value of the mass parameter $\mu^{2}$.

\subsection{Massive torsion waves}

For $\mu^{2}>0$, the characteristic functions $H$ and $K$ can be represented in the form

$$
H=y W_{0}, \quad K=W_{0},
$$

where $W_{0}$ is a generic wave "oscillatory" function,

$$
W_{0}:=C_{1}(u) \cos (m \ln y / \ell)+C_{2}(u) \sin (m \ln y / \ell) .
$$

In spite of this oscillatory behavior, both the torsion and the wave piece of the curvature tend to zero when $y \rightarrow 0$.

In the matrix notation, the components of the Siklos metric (2.1) read

$$
g_{\mu \nu}=\frac{\ell^{2}}{y^{2}}\left(\begin{array}{ccc}
2 H & 1 & 0 \\
1 & 0 & 0 \\
0 & 0 & -1
\end{array}\right) .
$$

Asymptotically, for $y \rightarrow 0$, we have $g_{u u} \sim W_{0} / y$, so that, to leading order in $1 / y, g_{\mu \nu}$ reduces to the AdS metric $\bar{g}_{\mu \nu}$. In the asymptotic analysis, we use $\mathcal{O}\left(y^{n} W_{0}\right)$ to denote a term that is at most proportional to $y^{n} W_{0}$ when $y \rightarrow 0$. Thus, the Siklos metric is of the type

$$
g_{\mu \nu}=\bar{g}_{\mu \nu}+G_{\mu \nu}, \quad G_{\mu \nu}:=\left(\begin{array}{cccc}
\mathcal{O}\left(W_{0} / y\right) & 0 & 0 \\
0 & 0 & 0 \\
0 & 0 & 0
\end{array}\right) .
$$

Looking at the action of the AdS Killing vectors (appendix A) on $g_{\mu \nu}$, one finds that the general requirements (a) and (b) are fulfilled by the following asymptotic configurations:

$$
g_{\mu \nu}=\bar{g}_{\mu \nu}+G_{\mu \nu}, \quad G_{\mu \nu}:=\left(\begin{array}{ccc}
\mathcal{O}_{-1} & \mathcal{O}_{0} & \mathcal{O}_{0} \\
\mathcal{O}_{0} & \mathcal{O}_{0} & \mathcal{O}_{0} \\
\mathcal{O}_{0} & \mathcal{O}_{0} & \mathcal{O}_{0}
\end{array}\right)
$$


where $\mathcal{O}_{n}:=\mathcal{O}\left(y^{n} W_{0}\right)$. The asymptotic form (4.2), but with $\mathcal{O}_{n}=\mathcal{O}\left(y^{n}\right)$, was studied earlier by Afshar et al. [34, 35], in the context of Conformal Chern-Simons gravity.

The asymptotic conditions (4.2) are preserved by the local translations of the form

$$
\begin{aligned}
\xi^{u} & =\varepsilon^{u}(u)+\frac{y^{2}}{4} \partial_{v}^{2} \varepsilon^{v}(v)+\mathcal{O}_{3}, \\
\xi^{v} & =\varepsilon^{v}(v)+\frac{y^{2}}{4} \partial_{u}^{2} \varepsilon^{u}(u)+\mathcal{O}_{3}, \\
\xi^{2} & =\frac{y}{2}\left(\partial_{u} \varepsilon^{u}+\partial_{v} \varepsilon^{v}\right)+\mathcal{O}_{3} .
\end{aligned}
$$

These parameters are essentially of the Brown-Henneaux type [35, 36].

In the next step, one could try to extend these considerations to the variables $b^{i}$ and $\omega^{i j}$. However, a problem arises when we return to our general requirement (c). Namely, although the field strengths $T^{i}$ and $R^{i j}$ have an AdS asymptotic limit, the asymptotic behavior of $b^{i}$ and $\omega^{i j}$ is determined by the function $W_{0}$, which oscillates when $y \rightarrow 0$. Thus, the basic dynamical variables have no asymptotic limit, and one is not able to define surface terms of the canonical generators. Thus, one cannot formulate a boundary theory, and in particular, the AdS/CFT correspondence is not well defined.

\subsection{Massless torsion waves}

In the sector with massless torsion modes, the form of our wave solution is displayed in eq. (3.10). As we noted before, the geometrically irrelevant term $D_{1}+D_{2} y^{2}$ in $H$ can be removed by choosing $D_{1}=D_{2}=0$, whereupon the characteristic functions $H$ and $K$ are of the generic form

$$
H=C_{0}(y / \ell), \quad K=C_{1}+C_{2} \ln (y / \ell) .
$$

The asymptotic geometry of the solution is described by the AdS spacetime. In this section, we discuss the asymptotic structure of the massless torsion wave (4.4).

Quite generally, the wave triad (2.2) can be written in the form $b^{i}{ }_{\mu}=\bar{b}^{i}{ }_{\mu}+B^{i}{ }_{\mu}$, where $\bar{b}^{i}$ is the AdS triad, and the only nonvanishing component of $B^{i}{ }_{\mu}$ is $B^{-}{ }_{u}=\ell H / y=C_{0}$. Then, in accordance with the general requirements (a) and (b), we choose the following asymptotic form of the triad field:

$$
b^{i}{ }_{\mu}=\bar{b}^{i}{ }_{\mu}+B^{i}{ }_{\mu}, \quad B^{i}{ }_{\mu}:=\left(\begin{array}{ccc}
\mathcal{O}_{1} & \mathcal{O}_{1} & \mathcal{O}_{1} \\
\mathcal{O}_{0} & \mathcal{O}_{1} & \mathcal{O}_{1} \\
\mathcal{O}_{1} & \mathcal{O}_{1} & \mathcal{O}_{1}
\end{array}\right)
$$

where $\mathcal{O}_{n}:=\mathcal{O}\left(y^{n}\right)$. These conditions impose the following restriction on the local Poincaré parameters $\left(\xi^{\rho}, \varepsilon^{i j}\right)$ :

$$
\delta_{0} b_{\mu}^{i}:=\varepsilon^{i j k} \theta_{j} b_{k \mu}-\left(\partial_{\mu} \xi^{\rho}\right) b_{\rho}^{i}-\xi^{\rho} \partial_{\rho} b^{i}{ }_{\mu}=B_{\mu}^{i},
$$

where $\theta^{i}$ is the Lie dual of $\varepsilon_{m n}$. As a consequence, the asymptotic parameters of local translations take the form displayed in eq. (4.3), whereas the asymptotic parameters of 
Lorentz rotations are found to be

$$
\begin{aligned}
\theta^{+} & =\frac{y}{2} \partial_{v}^{2} \varepsilon^{v}+\mathcal{O}_{2}, \\
\theta^{-} & =-\frac{y}{2} \partial_{u}^{2} \varepsilon^{u}+\mathcal{O}_{2}, \\
\theta^{2} & =\frac{1}{2}\left(\partial_{v} \varepsilon^{v}-\partial_{u} \varepsilon^{u}\right)+\mathcal{O}_{2} .
\end{aligned}
$$

Next, we wish to examine whether the asymptotic behavior of the RC connection (3.1) can be made compatible with the already found form of the asymptotic Poincaré parameters. First, we introduce the Lie-dual connection $\omega^{i}$ :

$$
\omega^{+}=\frac{1}{\ell} b^{+}, \quad \omega^{-}=-\frac{1}{\ell} b^{-}+\frac{y}{\ell} G b^{+}, \quad \omega^{2}=0 .
$$

The form of $K$ implies that the asymptotic conditions on the connection should contain $\log$ terms. By combining the expression (4.7) for $\omega^{i}{ }_{\mu}$ with the asymptotic formulas for $b^{ \pm}$ and $G=H^{\prime}+K$, we find it suitable to assume

$$
\omega^{i}{ }_{\mu}=\bar{\omega}^{i}{ }_{\mu}+\Omega^{i}{ }_{\mu}, \quad \Omega^{i}{ }_{\mu}:=\frac{1}{\ell}\left(\begin{array}{ll}
\mathcal{O}_{1} & \mathcal{O}_{1} \mathcal{O}_{1} \\
\mathcal{O}(\ln y / \ell) & \mathcal{O}_{1} \mathcal{O}(y \ln y / \ell) \\
\mathcal{O}(y \ln y / \ell) & \mathcal{O}_{1} \mathcal{O}_{1}
\end{array}\right)
$$

As it turns out, the asymptotic invariance of $\omega^{i}{ }_{\mu}$,

$$
\delta_{0} \omega_{\mu}^{i}:=-\partial_{\mu} \theta^{i}-\varepsilon^{i j k} \omega_{j \mu} \theta_{k}-\partial_{\mu} \xi^{\rho} \omega^{i}{ }_{\rho}-\xi^{\rho} \partial_{\rho} \omega^{i}{ }_{\mu}=\Omega_{\mu}^{i},
$$

does not impose any new restriction of the asymptotic Poincaré parameters (4.3) and (4.6).

In order to clarify the interpretation of our asymptotic conditions, we wish to find the commutator algebra of the asymptotic Poincaré transformations. To do that, we note that the composition law of the asymptotic transformations, to lowest order in $y$, reads

$$
\left(\varepsilon^{u}\right)^{\prime \prime \prime}=\left(\varepsilon^{u}\right)^{\prime} \partial_{u}\left(\varepsilon^{u}\right)^{\prime \prime}-\left(\varepsilon^{u}\right)^{\prime \prime} \partial_{u}\left(\varepsilon^{u}\right)^{\prime}
$$

and similarly for $\varepsilon^{v}$. Then, introducing the notation

$$
\ell_{n}^{+}:=-\frac{1}{\sqrt{2}} \delta_{0}\left(\varepsilon^{u}=\ell e^{i n u \sqrt{2} / \ell}, \varepsilon^{v}=0\right), \quad \ell_{n}^{-}:=-\frac{1}{\sqrt{2}} \delta_{0}\left(\varepsilon^{u}=0, \varepsilon^{v}=\ell e^{i n v \sqrt{2} / \ell}\right)
$$

the commutator algebra of the asymptotic symmetry takes the form of two independent Virasoro algebras:

$$
i\left[\ell_{m}^{ \pm}, \ell_{n}^{ \pm}\right]=(m-n) \ell_{m+n}^{ \pm} .
$$

The related central charges are discussed in the next section.

\section{Canonical form of the asymptotic symmetry}

In this section, we use the canonical approach to analyze the asymptotic symmetry in the massless sector, including the values of the central charges. 
To simplify the analysis, we follow Nester [37] in applying the first-order formulation to the quadratic PGT. In this formalism, the Lagrangian (3.4) is written in the form

$$
L_{G}=T^{i} \tau_{i}+\frac{1}{2} R^{i j} \rho_{i j}-V\left(b^{i}, \tau_{i}, \rho_{i j}\right)-\frac{1}{3} \Lambda \varepsilon_{i j k} b^{i} b^{j} b^{k} .
$$

Here, $\tau_{i}$ and $\rho_{i j}$ are new, independent variables, and $V$ is a function quadratic in $\tau_{i}$ and $\rho_{i j}$, chosen so that, on shell, we have $\tau_{i}=H_{i}$ and $\rho_{i j}=H_{i j}$, where $H_{i}=\partial L_{G} / \partial T^{i}$ and $H_{i j}=$ $\partial L_{G} / \partial R^{i j}$ are the covariant field momenta associated to the original Lagrangian (3.4). Explicit form of $V$ is described in ref. [31], and it ensures the first-order formulation (5.1) to be equivalent to (3.4). Thus, the variation of $L_{G}$ with respect to $\tau_{i}$ and $\rho_{i j}$ yields

$$
\begin{aligned}
\tau_{i} & =2 a_{1}^{\star} T_{i}, \\
\rho_{i j} & =-2\left(a_{0}-\frac{1}{6} b_{6} R\right) \varepsilon_{i j k} b^{k}+2 b_{4}{ }^{\star(4)} R_{i j},
\end{aligned}
$$

in accordance with the forms of $H_{i}$ and $H_{i j}$ defined by the Lagrangian (3.4).

Asymptotic symmetries are best described in the canonical formalism. In the first order formulation of PGT, the canonical gauge generator is a functional $G[\varphi, \pi]$ on the phase space, the form of which is defined in eqs. (5.7) of ref. [31]. The canonical generator acts on the phase-space variables $(\varphi, \pi)$ via the Poisson (or Dirac) bracket operation, defined in terms of the functional derivatives. A functional $F[\varphi, \pi]=\int d^{2} x f\left(\varphi, \partial_{\alpha} \varphi, \pi, \partial_{\alpha} \pi\right)$ is differentiable (or regular) if its variation has the form $\delta F=\int d^{2} x[A(x) \delta \varphi+B(x) \delta \pi]$. In order to ensure this property for our generator $G$, we have to improve its form by adding an appropriate surface term $\Gamma$ [38]. The improved canonical generator $\tilde{G}:=G+\Gamma$ has been calculated in appendix B; it is both finite and differentiable (well-defined).

The Poisson bracket (PB) algebra of the improved generators could be found by a direct calculation, but we rather rely on another, more instructive method. Introducing a convenient notation, $\tilde{G}^{\prime}=\tilde{G}\left[\varepsilon^{u \prime}, \varepsilon^{v \prime}\right]$ and similarly for $\tilde{G}^{\prime \prime}$ and $\tilde{G}^{\prime \prime \prime}$, we use the main theorem of ref. [39], which states that the PB of two well-defined generators must also be a well-defined generator, to conclude that the $\mathrm{PB}$ algebra has the form

$$
\left\{\tilde{G}^{\prime \prime}, \tilde{G}^{\prime}\right\}=\tilde{G}^{\prime \prime \prime}+C^{\prime \prime \prime}
$$

Here, the parameters of $\tilde{G}^{\prime \prime \prime}$ are defined by the composition law (4.9), and $C^{\prime \prime \prime}$ is the central charge of the algebra. A simple reformulation of this formula, given by

$$
\left\{\tilde{G}^{\prime \prime}, \tilde{G}^{\prime}\right\}=\delta_{0}^{\prime} \tilde{G}^{\prime \prime} \approx \delta_{0}^{\prime} \Gamma^{\prime \prime},
$$

represents a powerful tool for calculating the central charge. Indeed, the previous two equations imply

$$
\delta_{0}^{\prime} \Gamma^{\prime \prime} \approx \Gamma^{\prime \prime \prime}+C^{\prime \prime \prime}
$$

Now, since $C^{\prime \prime \prime}$ does not depend on the basic dynamical variables and $\Gamma^{\prime \prime \prime}$ vanishes on the AdS background (see appendix B), the evaluation of $\delta_{0}^{\prime} \Gamma^{\prime \prime}$ on the AdS background yields the final expression for $C^{\prime \prime \prime}$ :

$$
\overline{\delta_{0}^{\prime} \Gamma^{\prime \prime}}=C^{\prime \prime \prime}
$$


An explicit calculation based on the results of appendix B yields

$$
\frac{\sqrt{2}}{\ell} C^{\prime \prime \prime}=-\left(a_{0}-\frac{b_{6}}{\ell^{2}}\right) \int_{0}^{2 \pi} d \varphi\left(\varepsilon^{u \prime \prime} \partial_{u}^{3} \varepsilon^{u \prime}+\varepsilon^{v \prime \prime} \partial_{v}^{3} \varepsilon^{v \prime}\right)
$$

This result, combined with eq. (5.3a), completes the derivation of the canonical PB algebra.

A more familiar form of this algebra is obtained by introducing the Fourier modes of the improved generator:

$$
L_{n}^{+}:=-\frac{1}{\sqrt{2}} \tilde{G}\left(\varepsilon^{u}=\ell e^{i n u \sqrt{2} / l}, \varepsilon^{v}=0\right), \quad L_{n}^{-}:=-\frac{1}{\sqrt{2}} \tilde{G}\left(\varepsilon^{v}=\ell e^{i n v \sqrt{2} / l}, \varepsilon^{u}=0\right) .
$$

Then, the canonical algebra (5.3a) takes the form of two independent Virasoro algebras with central charges,

$$
i\left\{L_{m}^{ \pm}, L_{n}^{ \pm}\right\}=(m-n) L_{m+n}^{ \pm}+\frac{c^{ \pm}}{12} m^{3} \delta_{m+n},
$$

where the central charges are equal to each other:

$$
c^{ \pm}=\left(1-\frac{b_{6}}{a_{0} \ell^{2}}\right) c_{0} .
$$

Note that the coupling constant $b_{6}$ modifies the $\mathrm{GR}_{\Lambda}$ central charge $c_{0}:=3 \ell / 2 G$, and for $b_{6}<a_{0} \ell^{2}$, the central charge $c^{ \pm}$is positive.

\section{Concluding remarks}

In this paper, we found a new class of exact vacuum solutions of the three-dimensional PGT, the class of Siklos waves with torsion. Asymptotic geometry of these solutions is described by the Riemannian AdS spacetime. In the sector of massless torsion modes, we found a set of asymptotic conditions for which the asymptotic symmetry is described by two independent Virasoro algebras with equal central charges $c^{ \pm}$, the values of which differ from the $\mathrm{GR}_{\Lambda}$ result.

Further studies of the massless sector might help us to clarify the role of torsion in the AdS/CFT correspondence.

\section{Acknowledgments}

This work was supported by the Serbian Science Foundation under Grant No. 171031.

\section{A AdS and Siklos spacetimes in 3D}

In this appendix, we review basic aspects of the three-dimensional AdS and Siklos spacetimes; see for instance $[4,40,41]$ and $[7,8,16]$, respectively.

The AdS space in 3D, with topology $S^{1} \times R^{2}$, can be defined in terms of the hypersurface

$$
H_{3}: \quad \bar{u}^{2}-\bar{x}^{2}-\bar{y}^{2}+\bar{v}^{2}=\ell^{2},
$$


embedded in a 4-dimensional Minkowski space with metric $\eta_{a b}=(1,-1,-1,1)$. The metric on $H_{3}$ has the form

$$
d s^{2}=d \bar{u}^{2}-d \bar{x}^{2}-d \bar{y}^{2}+d \bar{v}^{2},
$$

its isometry group is $\mathrm{SO}(2,2)$, and the scalar curvature is $R=6 / \ell^{2}$.

The space $H_{3}$ can be covered by the global coordinates $(t, \rho, \varphi)$,

$$
\begin{array}{ll}
\bar{u}=\ell \cosh \rho \cos t, & \bar{x}=\ell \sinh \rho \cos \varphi, \\
\bar{v}=\ell \cosh \rho \sin t, & \bar{y}=\ell \sinh \rho \sin \varphi,
\end{array}
$$

with $t \in[-\pi, \pi], \rho \in[0, \infty)$, for which the metric takes the form

$$
d s^{2}=\ell^{2}\left[d t^{2} \cosh ^{2} \rho-\left(d \rho^{2}+\sinh ^{2} \rho d \varphi^{2}\right)\right] .
$$

However, since $t$ is an angle, there are closed timelike curves in $H_{3}$. The problem can be cured by replacing the $S^{1}$ time $t \in[-\pi, \pi]$ by a new, $R^{1}$ time $t \in(-\infty,+\infty)$, changing thereby the topology from $S^{1} \times R^{2}$ to $R^{3}$. The space obtained in this way is known as the universal covering of the AdS space. According to the commonly accepted terminology, it is this space that is called the AdS space; we denote it by $\mathrm{AdS}_{3}$. A simple form of the $\mathrm{AdS}_{3}$ metric is obtained in the Schwarzschild-like coordinates $r=\ell \sinh \rho, \ell t \rightarrow t$.

Let us now parametrize $\mathrm{AdS}_{3}$ by introducing the Poincaré coordinates:

$$
\tau=\frac{-\bar{v}}{\bar{u}+\bar{x}}, \quad x=\frac{\bar{y}}{\bar{u}+\bar{x}}, \quad y=\frac{\ell}{\bar{u}+\bar{x}} .
$$

They do not cover the whole space, but only one of the regions where $\bar{u}+\bar{x}$ has a definite sign. In these regions, the metric has the form

$$
d s^{2}=\frac{\ell^{2}}{y^{2}}\left(2 d u d v-d y^{2}\right)
$$

where $u=(\tau+x) / \sqrt{2}, v=(\tau-x) / \sqrt{2}$, and the boundary is located at $y=0$.

The Killing vectors $\xi=\xi^{\mu} \partial_{\mu}$ for the metric (A.3) are defined by the conditions

$$
\delta_{0} g_{\mu \nu}:=-\partial_{\mu} \xi^{\rho} g_{\rho \nu}-\partial_{\nu} \xi^{\rho} g_{\rho \mu}-\xi^{\rho} \partial_{\rho} g_{\mu \nu}=0 .
$$

They produce a set of requirements on $\xi^{\mu}$, the solutions of which define a basis of six independent AdS Killing vectors $\xi_{(m)}$ :

$$
\begin{array}{ll}
\xi_{(1)}=(\ell, 0,0), & \xi_{(4)}=(0,2 v, y), \\
\xi_{(2)}=(0, \ell, 0), & \xi_{(5)}=\left(\frac{u^{2}}{\ell}, \frac{y^{2}}{2 \ell}, \frac{u y}{\ell}\right), \\
\xi_{(3)}=(u,-v, 0), & \xi_{(6)}=\left(\frac{y^{2}}{2 \ell}, \frac{v^{2}}{\ell}, \frac{v y}{\ell}\right) .
\end{array}
$$

Turning now to the class of Siklos spacetimes (2.1), we note that it is equivalent to a subclass of Kundt spacetimes, defined by the metric

$$
d s^{2}=2\left(\frac{q}{p}\right)^{2} d U(\bar{H} d U+d V)-\frac{1}{p^{2}} d Y^{2},
$$


where $\bar{H}=\bar{H}(U, Y)$, and

$$
p:=1+\frac{\lambda}{4} Y^{2}, \quad q:=\left(1+\sqrt{-\frac{\lambda}{4}} Y\right)^{2}
$$

with $\lambda:=-1 / \ell^{2}$. Indeed, by introducing the new coordinates

$$
Y=-2 \ell \frac{y+1 / 2}{y-1 / 2}, \quad U=2 \ell u, \quad V=2 \ell v
$$

one ends up with the Siklos metric (2.1), where the new function $H=H(u, y)$ is defined by $H(u, y):=\left.\bar{H}(U, Y)\right|_{U=U(u), Y=Y(y)}$.

For general $H$, the only Killing vector of the Siklos metric is $\xi_{(2)}=\ell \partial_{v}$, but for some specific forms of $H$ there can be more Killing vectors; for instance, $\xi_{(1)}=\ell \partial_{u}$ when $H$ is independent of $u$, or the maximal number of six Killing vectors (A.4) when $H=0$.

\section{B Improving the canonical generator}

In this appendix, we construct the improved gauge generator for the massless sector of our solution.

Gauge symmetries of the first-order Lagrangian (5.1) are described by the canonical gauge generator $G$, the form of which can be found in eqs. (5.7) of ref. [31]. To examine the differentiability of $G$, we start from the form of its variation:

$$
\begin{aligned}
\delta G & =-\int_{\Sigma} d^{2} x\left(\delta G_{1}+\delta G_{2}\right) \\
\delta G_{1} & =-\varepsilon^{t \alpha \beta} \xi^{\mu}\left(b^{i}{ }_{\mu} \partial_{\alpha} \delta \tau_{i \beta}+\omega^{i}{ }_{\mu} \partial_{\alpha} \delta \rho_{i \beta}+\tau^{i}{ }_{\mu} \partial_{\alpha} \delta b_{i \beta}+\rho_{\mu}^{i}{ }_{\mu} \partial_{\alpha} \delta \omega_{i \beta}\right)+R \\
\delta G_{2} & =-\varepsilon^{t \alpha \beta} \theta^{i} \partial_{\alpha} \delta \rho_{i \beta}+R .
\end{aligned}
$$

Here, the variation is performed in the set of asymptotic states, $R$ stands for regular terms and $\rho^{i}$ is the Lie dual of $\rho_{m n}$ :

$$
\rho_{i}=2\left(a_{0}-\frac{b_{6}}{6} R\right) b_{i}+2 b_{4}\left((R i c)_{(i k)}-\frac{1}{3} R \eta_{i k}\right) b^{k} .
$$

Moreover, the coherently oriented volume 2-form on $\Sigma$, expressed in the new coordinates $(t, \varphi, y)$, is normalized to $d^{2} x=d y d \varphi$. Together with $\varepsilon^{y \varphi}:=\varepsilon^{t y \varphi}=1$, this is in accordance with the conventions used in ref. [31].

As one can see, $G$ is not differentiable, but the problem can be corrected by going over to the improved canonical generator $\tilde{G}:=G+\Gamma$, where the surface term $\Gamma$ is constructed so that $\delta \tilde{G}=R$. In the process, transition to surface integrals is performed with the help of the Stokes formula:

$$
\int_{\Sigma} d^{2} x \partial_{\alpha} v^{\alpha}=\int_{\partial \Sigma} d f_{\alpha} v^{\alpha}=\int_{0}^{2 \pi} d \varphi v^{y}, \quad d f_{\alpha}=\varepsilon_{\alpha \beta} d x^{\beta}
$$


Thus, using (B.1) and the asymptotic conditions (4.5) and (4.8), the surface term $\Gamma$ in the improved generator $\tilde{G} \equiv G+\Gamma$ is found to have the following form:

$$
\begin{aligned}
\Gamma= & \Gamma_{u}+\Gamma_{v} \\
\frac{\sqrt{2}}{\ell} \Gamma_{u}= & -2\left(a_{0}-\frac{b_{6}}{\ell^{2}}\right) \int_{0}^{2 \pi} d \varphi \epsilon^{u} \frac{1}{y}\left(B^{-}{ }_{u}-B^{-}{ }_{v}\right)+2 \ell a_{1} \int_{0}^{2 \pi} d \varphi \epsilon^{u} \partial_{y}\left(B^{-}{ }_{u}-B^{-}{ }_{v}\right) \\
& +\frac{2 b_{4}}{\ell} \int_{0}^{2 \pi} d \varphi \epsilon^{u}\left(\partial_{y} \Omega^{-}{ }_{u}-\partial_{u} \Omega^{-}{ }_{y}+\frac{1}{y} \frac{B^{-} u}{\ell}\right) \\
\frac{\sqrt{2}}{\ell} \Gamma_{v}= & 2\left(a_{0}-\frac{b_{6}}{\ell^{2}}\right) \int_{0}^{2 \pi} d \varphi \epsilon^{v} \frac{\ell}{y}\left({\Omega^{+}}_{u}-{\Omega^{+}}_{v}+\frac{1}{\ell} B^{+}{ }_{u}-\frac{1}{\ell} B^{+} v\right) .
\end{aligned}
$$

The result for $\Gamma_{u}$ is simplified with the help of the condition $a_{0}-b_{6} / \ell^{2}-a_{1}=0$, which is used in eq. (3.9) to define the massless sector of the torsion wave. The factors $\sqrt{2} / \ell$ appear as an effect of the change of coordinates $(t, \varphi) \rightarrow(u, v)$ in the components of $B^{i}$ and $\Omega^{i}$.

The above construction shows that $\tilde{G}$ is differentiable provided it is finite, and the finiteness of $\tilde{G}$ follows from the finiteness of $\Gamma \equiv \Gamma_{u}+\Gamma_{v}$. The term $\Gamma_{v}$ is seen to be finite directly from the adopted asymptotic conditions, whereas the finiteness of $\Gamma_{u}$ depends on the validity of an additional relation:

$$
-\left(a_{0}-\frac{b_{6}}{\ell^{2}}-\frac{b_{4}}{\ell^{2}}\right) B^{-}{ }_{u}+\frac{b_{4}}{\ell} y \partial_{y} \Omega^{-}{ }_{u}=\mathcal{O}_{1} .
$$

To clarify this situation, we note that the original set of the asymptotic conditions, given in eqs. (4.5) and (4.8), can be extended using the following general principle: the expressions that vanish on-shell should have an arbitrarily fast asymptotic decrease, as no solution of the field equations is thereby lost. This principle allow us to derive the needed relation (B.3) as the $(\mu=v, i=+)$ component of the field equation

$$
\varepsilon^{\mu \nu \rho}\left(\nabla_{\mu} \rho_{i \nu}+\varepsilon_{i j k} b_{\nu}^{j} \tau_{\rho}^{k}\right)=0 .
$$

The surface terms (B.2) are used in section 5 to calculate the canonical algebra of the improved gauge generators. Note, in particular, that $\Gamma$ vanishes on the AdS background.

Open Access. This article is distributed under the terms of the Creative Commons Attribution License (CC-BY 4.0), which permits any use, distribution and reproduction in any medium, provided the original author(s) and source are credited.

\section{References}

[1] J. Ehlers and W. Kundt, Exact solutions of the gravitational field equations, in Gravitation: an Introduction to Current Research, L. Witten eds., Willey, New York U.S.A. (1962), pg. 49-101.

[2] V. Zakharov, Gravitational Waves in Einstein's Theory, Halsted Press, New York U.S.A. (1973).

[3] H. Stephani, D. Kramer, M. MacCallum, C. Hoenselaers and E. Herlt, Exact Solutions of Einstein's Field Equations, second edition, Cambridge University Press, Cambridge U.K. (2003). 
[4] J.B. Griffiths and J. Podolský, Exact Space-Times in Einstein's General Relativity, Cambridge University Press, New York U.S.A. (2009).

[5] A. García Díaz and J.F. Plebański, All nontwisting N's with cosmological constant, J. Math. Phys. 22 (1981) 2655.

[6] H.I. Salazar, A. García Díaz and J.F. Plebański, Symmetries of the nontwisting type-N solutions with cosmological constant, J. Math. Phys. 24 (1983) 2191.

[7] I. Ozsváth, I. Robinson and K. Rózga, Plane fronted gravitational and electromagnetic waves in spaces with cosmological constant, J. Math. Phys. 26 (1985) 1755 [INSPIRE].

[8] J. Bičák and J. Podolský, Gravitational waves in vacuum space-times with cosmological constant. 1. Classification and geometrical properties of nontwisting type $N$ solutions, J. Math. Phys. 40 (1999) 4495 [gr-qc/9907048] [INSPIRE].

[9] Y.N. Obukhov, Generalized plane fronted gravitational waves in any dimension, Phys. Rev. D 69 (2004) 024013 [gr-qc/0310121] [InSPIRE].

[10] A. Coley, R. Milson, N. Pelavas, V. Pravda, A. Pravdova and R. Zalaletdinov, Generalizations of pp-wave spacetimes in higher dimensions, Phys. Rev. D 67 (2003) 104020 [INSPIRE].

[11] V.P. Frolov and A. Zelnikov, Relativistic gyratons in asymptotically AdS spacetime, Phys. Rev. D 72 (2005) 104005 [INSPIRE].

[12] J. Podolský and M. Zofka, General Kundt spacetimes in higher dimensions, Class. Quant. Grav. 26 (2009) 105008 [arXiv:0812.4928] [INSPIRE].

[13] M. Ortaggio, V. Pravda and A. Pravdova, Algebraic classification of higher dimensional spacetimes based on null alignment, Class. Quant. Grav. 30 (2013) 013001 [arXiv:1211.7289] [INSPIRE].

[14] E. Witten, Anti-de Sitter space and holography, Adv. Theor. Math. Phys. 2 (1998) 253 [hep-th/9802150] [INSPIRE].

[15] O. Aharony, S.S. Gubser, J. Maldacena, H. Ooguri and Y. Oz, Large $N$ field theories, string theory and gravity, Phys. Rept. 323 (2000) 183 [hep-th/9905111] [INSPIRE].

[16] J. Podolský, Interpretation of the Siklos solutions as exact gravitational waves in the anti-de Sitter universe, Class. Quant. Grav. 15 (1998) 719 [gr-qc/9801052] [INSPIRE].

[17] S. Carlip, Quantum Gravity in 2+1 Dimensions, Cambridge University Press, Cambridge U.K. (1998).

[18] S. Carlip, Conformal field theory, (2+1)-dimensional gravity and the BTZ black hole, Class. Quant. Grav. 22 (2005) R85 [gr-qc/0503022] [InSPIRE].

[19] S. Deser, R. Jackiw and S.-Y. Pi, Cotton blend gravity pp waves, Acta Phys. Polon. B 36 (2005) 27 [gr-qc/0409011] [INSPIRE].

[20] E. Ayón-Beato and M. Hassaïne, Scalar fields nonminimally coupled to pp waves, Phys. Rev. D 71 (2005) 084004 [hep-th/0501040] [INSPIRE].

[21] E. Ayón-Beato and M. Hassaïne, Exploring AdS waves via nonminimal coupling, Phys. Rev. D 73 (2006) 104001 [hep-th/0512074] [INSPIRE].

[22] S. Deser, R. Jackiw and S. Templeton, Three-Dimensional Massive Gauge Theories, Phys. Rev. Lett. 48 (1982) 975 [INSPIRE]. 
[23] S. Deser, R. Jackiw and S. Templeton, Topologically Massive Gauge Theories, Annals Phys. 140 (1982) 372 [Erratum ibid. 185 (1988) 406] [INSPIRE].

[24] E.A. Bergshoeff, O. Hohm and P.K. Townsend, Massive Gravity in Three Dimensions, Phys. Rev. Lett. 102 (2009) 201301 [arXiv:0901.1766] [INSPIRE].

[25] E. Ayón-Beato, G. Giribet and M. Hassaïne, Bending AdS Waves with New Massive Gravity, JHEP 05 (2009) 029 [arXiv:0904.0668] [INSPIRE].

[26] M. Blagojević, Gravitation and Gauge Symmetries, Institute of Physics Publishing, Bristol U.K. (2002).

[27] T. Ortín, Gravity and Strings, Cambridge University Press, Cambridge U.K. (2004).

[28] M. Blagojević and F. W. Hehl eds., Gauge Theories of Gravitation, A Reader with Commentaries, Imperial College Press, London U.K. (2013).

[29] Y.N. Obukhov, Poincaré gauge gravity: selected topics, Int. J. Geom. Meth. Mod. Phys. 3 (2006) 95 [gr-qc/0601090] [INSPIRE].

[30] Y.N. Obukhov, New solutions in 3D gravity, Phys. Rev. D 68 (2003) 124015 [gr-qc/0310069] [INSPIRE].

[31] M. Blagojević and B. Cvetković, 3D gravity with propagating torsion: the AdS sector, Phys. Rev. D 85 (2012) 104003 [arXiv:1201.4277] [InSPIRE].

[32] M. Blagojević and B. Cvetković, Gravitational waves with torsion in 3D, Phys. Rev. D 90 (2014) 044006 [arXiv: 1406.2850] [INSPIRE].

[33] S.T.C. Siklos, Lobatchevski plane gravitational waves, in Galaxies, Axisymmetric Systems and Relativity, M.A.H. MacCallum eds., Cambridge University Press, Cambridge U.K. (1985), pg. 247-274.

[34] H. Afshar, B. Cvetković, S. Ertl, D. Grumiller and N. Johansson, Holograms of Conformal Chern-Simons Gravity, Phys. Rev. D 84 (2011) 041502 [arXiv: 1106.6299] [inSPIRE].

[35] H. Afshar, B. Cvetković, S. Ertl, D. Grumiller and N. Johansson, Conformal Chern-Simons holography - lock, stock and barrel, Phys. Rev. D 85 (2012) 064033 [arXiv:1110.5644] [INSPIRE].

[36] A. Strominger, A Simple Proof of the Chiral Gravity Conjecture, arXiv:0808.0506 [INSPIRE].

[37] J.M. Nester, A covariant Hamiltonian for gravity theories, Mod. Phys. Lett. A 6 (1991) 2655 [INSPIRE].

[38] T. Regge and C. Teitelboim, Role of Surface Integrals in the Hamiltonian Formulation of General Relativity, Annals Phys. 88 (1974) 286 [INSPIRE].

[39] J.D. Brown and M. Henneaux, On the Poisson Brackets of Differentiable Generators in Classical Field Theory, J. Math. Phys. 27 (1986) 489 [inSPIRE].

[40] S. Hawking and G. Ellis, The Large Scale Structure of Spacetime, Cambridge University Press, Cambridge England (1973).

[41] M. Bañados, M. Henneaux, C. Teitelboim and J. Zanelli, Geometry of the (2+1) black hole, Phys. Rev. D 48 (1993) 1506 [gr-qc/9302012] [INSPIRE]. 